\title{
Using wireless acceleration sensor for system identification
}

\author{
Peter Šarafín \\ University of Žilina \\ Faculty of Management Science and Informatics, \\ Univerzitná 8215/1, Žilina 01026 \\ Email: peter.sarafin@fri.uniza.sk
}

\author{
Juraj Miček, Jana Milanová \\ University of Žilina \\ Faculty of Management Science and Informatics, \\ Univerzitná 8215/1, Žilina 01026 \\ Email: \{juraj.micek, jana.milanova\}@fri.uniza.sk
}

\begin{abstract}
In practical applications, we often encounter problems controlling weakly damped resonant systems. These are devices which often include inertia masses and flexible connecting elements. These devices are mainly gantry cranes, mechatronic systems, elevators, filling lines for the food industry and many others. One approach to improving the transition process in the control of these weakly damped systems is a method of shaping control signals. This method starts to be used in the control of systems with flexible elements in the 90s of the twentieth century. Over the next twenty years, we meet with successful applications, especially in the control of positioning systems. When we are talking about the theoretical description of input shaping today, we meet mainly with two basic approaches. The first is based on the selection of a proper sequence of pulses in the time domain. The second is based on the design of such discrete shaper, which compensates the effect of the complex poles of a controlled system causing residual vibration. Irrespective of the shaper design method, we must know either the systems oscillations and controlled system damping, or the location of the complex poles causing the vibration.
\end{abstract}

\section{INTRODUCTION}

$\mathbf{T}$ HE AIM of the input shaper is to adjust the control signals of the weakly damped system in order to eliminate residual vibrations of the system. With its proposal, we try to minimize transition time simultaneously. To illustrate this, let us have oscillatory system with the transfer function:

$$
\left.F(s)=\frac{1}{T^{2} s^{2}+2 b T s+1}, b \in<0,1\right),
$$

where $b$ is the damping of the system and $T$ is the time constant.

The task is to propose such methods of the control signal adjustment, that the transition process fits stated requirements. The possible system arrangement is shown in figure 1. It is evident that the shaper acts as a serial correction element that is known from the classical theory of automated control.

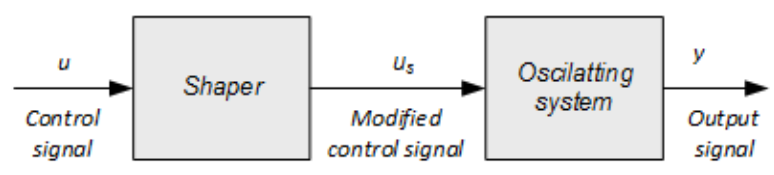

Fig. 1. The input shaping process
For the shaping of the control signal, two approaches can be used:

1) Signal shaping in the time domain, e.g. direct generation of the control signals with suitable properties [1], [2].

2) Using discrete shaping members [6], [9].

Appropriately chosen shaper suppress residual vibrations of the system. Note that the suppression ratio of residual vibrations is often expressed as the ratio of the amplitude of the output signal with the shapers input signal to the amplitude of the output signal without the shaper [4], [7], [8].

\section{PROPOSAL OF AN INPUT SHAPER IN THE TIME DOMAIN}

The rate of suppression of residual vibrations can be expressed as a function of the angular velocity $\omega$ and the proportional damping factor $\mathrm{b}$ of the system as:

$$
V(\omega, b)=e^{-b \omega t_{n}} \sqrt{(C(\omega, b))^{2}+(S(\omega, b))^{2}},
$$

while:

$$
\begin{aligned}
& C(\omega, b)=\sum_{i=1}^{n} A_{i} \cdot e^{b \omega t_{i}} \cdot \cos \left(\omega \cdot t_{i} \sqrt{1-b^{2}}\right), \\
& S(\omega, b)=\sum_{i=1}^{n} A_{i} \cdot e^{b \omega t_{i}} \cdot \sin \left(\omega \cdot t_{i} \sqrt{1-b^{2}}\right),
\end{aligned}
$$

where $A_{i}$ is the amplitude, $t_{i}$ is the time of occurrence of the ith pulse and $n$ is the number of input shapers pulses. Ideally, with complete suppression of the vibrations, the relation (2) has to be equal to zero for its own circular speed and relative system damping. It is obvious that for $\omega=\omega_{0}$ is given:

$$
C\left(\omega_{0}\right)=0, S\left(\omega_{0}\right)=0,
$$

If the shaped output is to have the same final value as the unshaped (normalized shaper), then it is given that the sum of the amplitudes of all pulses has to be equal to one:

$$
\sum_{i=1}^{n} A_{i}=1 .
$$

Note that if the pulse amplitudes $A_{i}$ are not limited, then in terms of minimizing the total time of transition $t_{n}$, it will acquire an infinitely large value. When practical solutions, we most often encounter with two restrictive conditions: 
3) The pulse amplitudes can take values from the range of $\pm A_{\max }$

4) The pulse amplitudes can take only non-negative values $A_{i} \geq 0$.

It is obvious that if amplitudes of all the pulses are nonnegative, then respecting conditions (5), it must belong to the interval $0 \leq A i \leq 1$. With respect to those limitations, by solving the equations (2) we get for $\min \left(t_{n}\right)$ the solution describing positive $\mathrm{ZV}$ shaper whose design is often stated in matrix form [3], [5], [6], [7]:

$$
\left[\begin{array}{c}
A_{i} \\
t_{i}
\end{array}\right]=\left[\begin{array}{cc}
\frac{1}{1+K} & \frac{K}{1+K} \\
0 & 0.5 \cdot T_{D}
\end{array}\right]
$$

where:

$$
T_{D}=\frac{2 \cdot \pi \cdot T}{\sqrt{1-b^{2}}}, K=e^{-\frac{b \pi}{\sqrt{1-b^{2}}}} .
$$

ZV shaper (zero-vibration) (6) may be described in the time domain by a relation:

$$
y(t)=A_{1} \cdot \delta(t)+A_{2} \cdot \delta\left(t-t_{2}\right) .
$$

$\mathrm{ZV}$ shaper described, however, is quite sensitive to changes in the parameters of the controlled system, in particular, to the changes in its own circular speed. For this reason, more robust input shapers have been developed. The most famous is ZVD (zero vibration derivate) shaper. In deriving the ZVD shaper, it is assumed that the derivative of the function $V(\omega, b)$ is equal to zero.

$$
\left[\begin{array}{c}
A_{i} \\
t_{i}
\end{array}\right]=\left[\begin{array}{ccc}
\frac{1}{(1+K)^{2}} & \frac{2 K}{(1+K)^{2}} & \frac{K^{2}}{(1+K)^{2}} \\
0 & 0.5 \cdot T_{D} & T_{D}
\end{array}\right]
$$

From (9) it is clear that the increase of robustness is penalized by the increase of the transition time. Transition time rose from $0.5 \cdot T_{D}$ to $T_{D}$.

\section{PROPOSAL OF DISCRETE INPUT SHAPER}

The problem of suppression of residual vibrations in the control of weakly damped systems can be successfully resolved with the appropriate design of discrete systems (correction elements) regulating the spectrum of the control signal to suppress residual vibration of the system. It is clear that the task can be solved by the appropriate placing the zeros of the $\mathrm{z}$-transfer function characteristic element to the z-plane points corresponding to the field effect system. As the controlled system is characterized by a continuous transfer function, it is necessary to find a suitable transformation of the continuous system to a discrete equivalent. The discrete shaper will then be the inverse of the discrete equivalent of the continuous system. If the designed shaper compensates only selected complex transfer poles, then it is sufficient to place zeroes to the appropriate poles of the shaper. To find the discrete equivalent of the continuous system $F(s)$ defined by poles which cause oscillation of the system, it is suitable to use the transform between $\mathrm{s}$ and $\mathrm{z}$ plane by using the relation:

$$
z=e^{s T_{v}}
$$

where $T_{v}$ is the sampling period.

Recall that we want to compensate only complex poles, therefore we consider the transfer in the form:

$$
F(s)=\frac{1}{\prod_{i=1}^{N}\left(T_{i}^{2} s^{2}+2 b_{i} T_{i} s+1\right)},
$$

where $N$ represents the number of pairs of complex conjugate poles to be compensated, $T_{i}$ stands for the time constant and $b_{i}$ is the damping coefficient of the ith subsystem. The poles of the system with transfer (11) are:

$$
p_{i, 1,2}=-\frac{b_{i}}{T_{i}} \pm j \frac{\sqrt{1-b^{2}}}{T}, i=1,2,3, \ldots, N
$$

In applying the transformation equation (10), for the poles of discrete equivalent of continuous system (11) will apply:

$$
p_{d i, 1,2}=r_{i} e^{ \pm j \phi_{i}}=e^{-\frac{T_{v} b_{i}}{T_{i}}} e^{ \pm j \frac{T_{v} \sqrt{1-b_{i}^{2}}}{T_{i}}}, i=1,2, \ldots, N
$$

To compensate for the effect of selected poles of the continuous system, the discrete shaper has to contain zeroes in such points in the z-plane that correspond to poles of the discrete equivalent $\left(p_{i 1,2}\right)$ (Fig. 2).

If we place the zeroes of the shapers $\mathrm{z}$-transfer function into the points corresponding to the position of poles, then transfer function shaper will be in the form:

$$
F(z)=\prod_{i=1}^{N}\left(z-z_{i 1}\right)\left(z-z_{i 2}\right)
$$

alternatively, when considering a $2 \mathrm{~N}$-multiple pole at the origin of the plane:

$$
\begin{gathered}
F(z)=\prod_{i=1}^{N} C_{i}\left(1-z_{i 1} z^{-1}\right)\left(1-z_{i 2} z^{-1}\right) \\
F(z)=\prod_{i=1}^{N} C_{i}\left(a_{i 2} z^{-2}+a_{i 1} z^{-1}+a_{i 0}\right)
\end{gathered}
$$

where

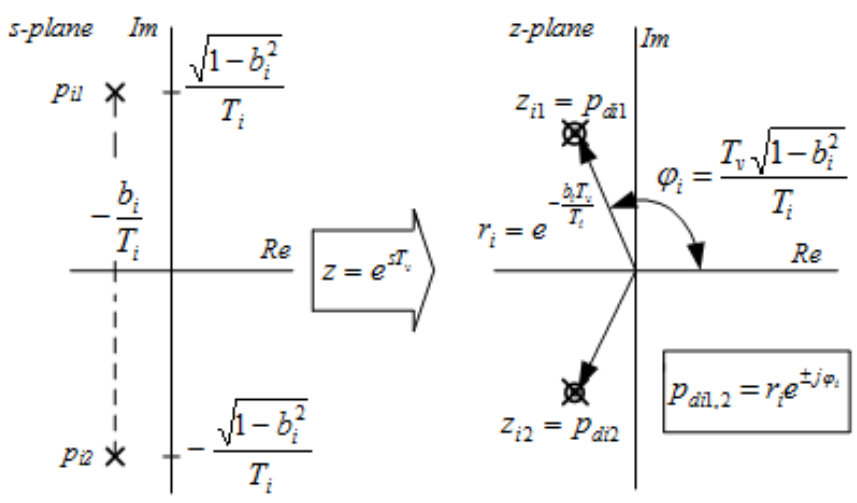

Fig. 2. Poles representation in the s-plane and z-plane 


$$
\begin{aligned}
C_{i} & =\prod_{i=1}^{N} \frac{1}{a_{i 0}+a_{i 1}+a_{i 2}}, \\
a_{i 0} & =1, a_{i 1}=-\left(z_{1}+z_{2}\right), a_{i 2}=z_{1} \cdot z_{2},
\end{aligned}
$$

alternatively:

$$
\begin{array}{r}
a_{i 0}=1, a_{i 1}=-2 r_{i} \cos (\phi i), a_{i 2}=r_{i}^{2}, \\
r_{i}=e^{-\frac{T_{v i} b_{i}}{T_{i}}}, \phi_{i}=\frac{T_{v i} \sqrt{1-b_{i}^{2}}}{T_{i}} .
\end{array}
$$

When defining the gain $C_{i}$, we assume that consistentely with the continuous system transfer(11) is the shaper normalized (shaper gain is equal to one). From (13) it is clear that the position of poles of the discrete equivalent of the continuous system (11) $p_{d i, 1,2}$ can be changed in the z-plane by selecting the sampling period $T_{v}$. This is illustrated in figure 3 .

The curves on the right side of figure 3 illustrate a possible $p_{d 1,2}$ poles location of the discrete equivalent of a continuous system, depending on the selection of sampling period $T_{v}$. The left side of figure 3 shows the poles of the original continuous oscillating system with the transfer function (1). It is clear that the value $T_{v}$ can be theoretically chosen in the range of 0 to $\infty$. Recall that the settling time of system output is proportional to the sampling period $T_{v}$. It follows that if we focus on achieving good system dynamics shaper-system, we are trying to make $T_{v}$ minimal during the design of the shaper. Obviously, the choice of $T_{v}$ minimum value is related to the energy options of the actuator and the constraints of system input. More specifically, the input shaper synthesis and the problem of choice of sampling frequency was discussed in the paper [10].

\section{THE PROBLEM OF OSCILLATING SUBSYSTEM IDENTIFICATION}

As already indicated, the basic assumption of the successful suppression of residual vibrations of a transition process by input shaper is the knowledge of the controlled oscillating subsystem parameters. Due to the rapid adaptation, it is advisable to devote to the identification of only that part of the system, which causes vibration. The proposed solution is illustrated in figure 4. This example does not discuss the

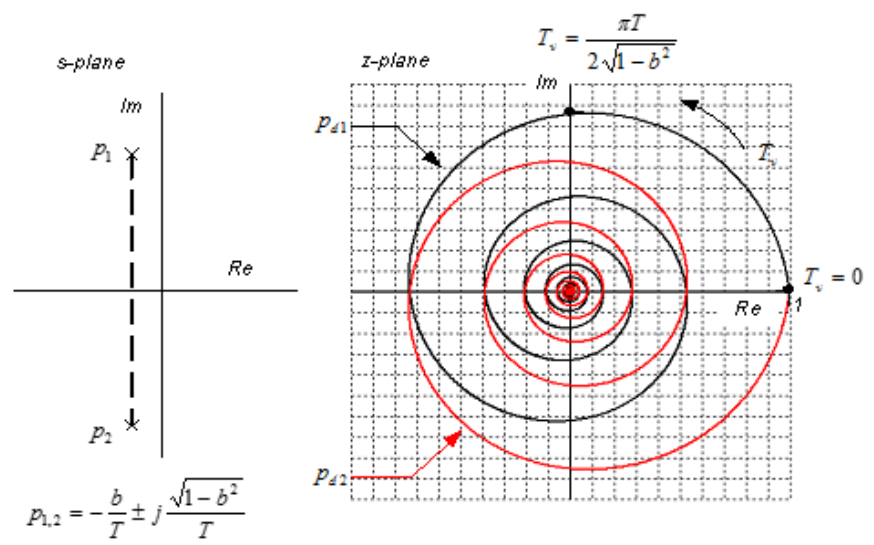

Fig. 3. Locus of the poles of discrete equivalent of the continuous system identification of the drive of subsystem $M$, but only analyze the dependence of the pivot position $x(t)$ and the load position $y(t)$.

In order to verify the proposed comprehensive solutions, wireless acceleration sensors have been developed in our department. The sensors were used to measure the displacement $x(t)$ and the load movement $y(t)$. Based on the input to the oscillating system section $x(t)$ and the corresponding output $y(t)$, parameters $T$ and $b$ of the oscillating section were identified with the transfer function:

$$
\begin{gathered}
F(s)=\frac{1}{T^{2} s^{2}+2 b T s+1} \\
\text { V. CASE STUDY }
\end{gathered}
$$

In general, it is common to face to the problem of identification in control applications. In order to be able to appropriately modify the control signal and thus the system response to this signal, it is important to know the transfer function of the system. For this purpose, the modules providing collection and data transfer were designed.

\section{A. Description of used nodes}

When designing the node, microcontroller ATmega168 was chosen as an element controlling the data collection and transfer. Considering that the system frequency and damping have to be known in the identification, we decided to retrieve data through LSM303DLHC module that includes a 3D digital linear acceleration sensor and a 3D digital magnetic sensor. Data obtained from the accelerometer is sent via I2C serial bus interface to the microcontroller. The microcontroller uses a timer/counter for timing each data collection process, and it ensures equidistance sampling $(f s=400 H z)$. The output of the accelerometer is represented by three 16-bit words, where each represents one of the axes $\mathrm{x}, \mathrm{y}$, and $\mathrm{z}$. Gathered data is further sent through the wireless module RFM70. The proprietary module operates at a $2.4 \mathrm{GHz}$ frequency, and can be configured as a transmitter and as a receiver. Communication with RFM70 is ensured via the SPI interface. The data from the nodes is sent at each subsequent reading of the accelerometer, which means that we have a real-time data.

In order to get the data to the PC for processing, the node consisting of a microcontroller ATmega8 was proposed. Its role is to ensure the data reception from two transmitters via wireless module RFM70 and subsequently to send the data to

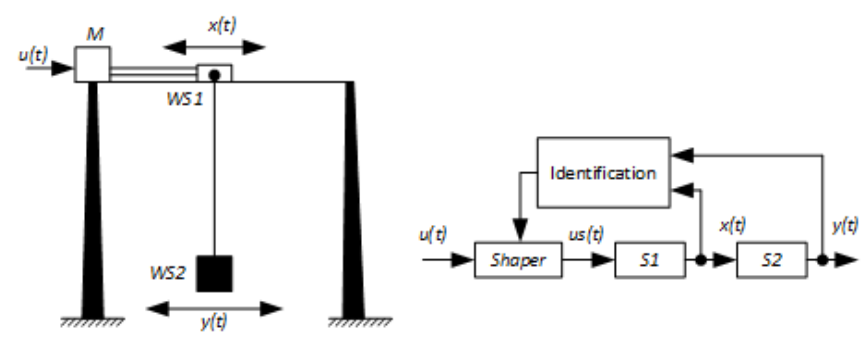

Fig. 4. Oscillating subsystem identification 


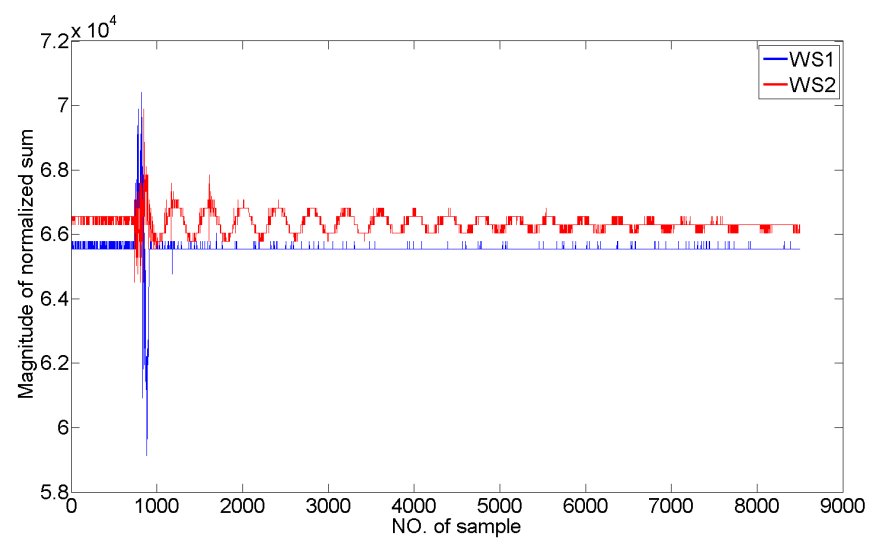

Fig. 5. Step response of weakly damped system

the PC via the UART interface. Here the data is visualized and further processed.

\section{B. The measured results}

Data obtained from the 3D accelerometers is sent via the RS232 interface to the PC where it is rendered over Matlab environment in the graph in real-time. In addition to displaying operation, the received data is recorded to the file, which forms the basis for further processing. Considering the change of the sensor position is not recorded only in one accelerometer axis, the data measured at the same time is summed. The data obtained through sensors $W S 1$ and $W S 2$ is represented in figure 5 .

\section{System identification}

In order to identify the system, it is important to describe the output signal. As can be seen in figure 5, the output signal has damped oscillatory character after excitation (Fig. 6) [11]. As the input signal, the output signal is stabilized at the original level.

From the data obtained, it is approximated (determined) the system equation. After the system excitation, we get the local minimum $K-y_{2}$ and the local maximum $y_{1}-K$. These values are in equation 19 represented as $x_{1}$ and $x_{2}$.

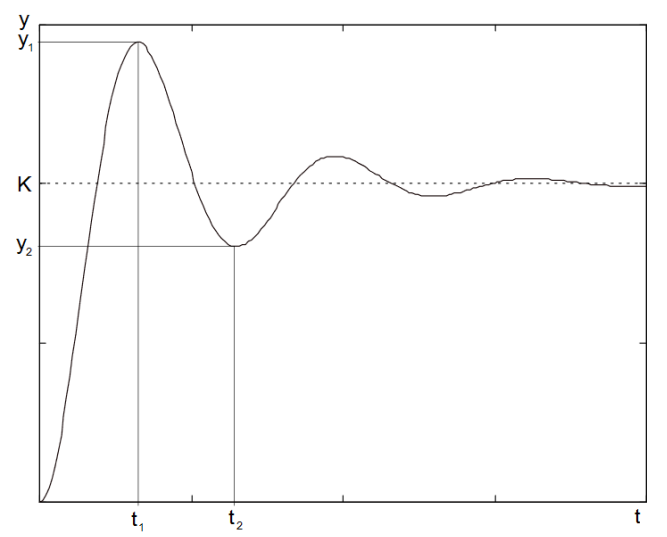

Fig. 6. Step response of weakly damped system

$$
b=\ln \left(\frac{x_{1}}{x_{2}}\right) \cdot \frac{2}{T},
$$

where $b$ represents the damping of the system output. Period $T$, and thus the natural frequency of the system is defined by a difference between the times when there was a local minimum $t_{2}$ and the local maximum $t_{1}$. Substituting the obtained parameters in equation 18, we get the system step response (Eq. 20).

$$
\begin{gathered}
F(s)=\frac{1}{0.98^{2} s^{2}+2 \cdot 0.1702 \cdot 0.98 s+1} \\
\text { VI. CONCLUSION }
\end{gathered}
$$

In this paper, conventional methods of input shaping techniques were reviewed. To propose suitable input shaper the controlled system should be identified. For this reason, nodes providing data from the accelerometer and wireless communication were constructed. These devices are small so they can be used also in other applications (e. g. to identify weaknesses of construction proposal and getting its mathematical model).

As the results show, the identification of second order system is not computationally demanding but captured data suffer from unwanted noise. To improve the process of identification data gathered from the accelerometer should be appropriately filtered.

There is a need to propose new nodes involving the gyroscope coming to the fore. This fact will result in the future work that will be focused on data fusion, particularly combining data from accelerometer and gyroscope.

\section{REFERENCES}

[1] J. Fortgang, V. Patrangenaru, W. Singhose, " Scheduling of Input Shaping and Transient Vibration Absorbers for High-Rise Elevators", in Proceedings of the American Control Conference, 2006

[2] N. C. Singer, W. Seering, "Per shaping Command Inputs to reduce Systems Vibration, Journal of Dynamic Systems“, in Measurement and Control, vol. 112, 1990

[3] P.Hubinský, P. Hauptle, "Reducing Oscillation during Positioning of a Servomechanism having Flexibility“, in Journal of Electrical Engineering Vol.63, No.4, 2012, ISSN 1335-3632

[4] M. J. Robertson, W. E. Singhose, "Multi-Level Optimization Techniques for Designing Digital Input Shapers", in Proceedings of the American Control Conference, Arlington 2001

[5] E. Biediger, J. Lawrence, W. Singhose, "Improving Trajectory Tracking for Systems with Unobservable Modes Using Command Generation", in Proceedings of the American Control Conference, Portland 2005

[6] L. Y. Pao, C. F. Cutforth, "On Frequency-Domain and Time-Domain input Shaping for Multi-Mode Flexibile Structures“, in ASME vol. 125, 2003

[7] N. Singer, W. Singhose, W. Seering, "Comparison of Filtering Methods for Reducing Residual Vibration", in European Journal of Control, 1999

[8] K. Kozak, J. H uey, W Sighose, "Performance Measures for Input Shaping", in Proceedings of the IEEE Conf. on Control Applications, Istanbul, Turkey, 2003

[9] T. D. Tuttle, W. P. Seering, "A Zero-placement Technique for Designing Shaped Inputs to Suppress Multiple-mode Vibration", in Proceedings of the American Control Conference, Baltimore 1994

[10] J. Miček, J. Juríček, "Discrete shaper of control signals", in International journal of engineering research in Africa. - ISSN 1663-3571. - Vol. 18 (2015), s. 65-74

[11] M. Fikar, J. Mikleš, "Identifikácia systémov", in https://www.kirp.chtf. stuba.sk/fikar/research/ident/ident.pdf - ISBN 80-227-1177-2. 